\title{
Agent-based Traffic Operator Training Environments for Evacuation Scenarios
}

\author{
David Handford and Alex Rogers \\ Electronics and Computer Science \\ University of Southampton \\ Southampton, UK \\ Email: $\{$ djh07r,acr\}@soton.ac.uk
}

\author{
Kevin Cross \\ BAE Systems \\ Filton, Bristol, UK \\ Email: Kevin.J.Cross@baesystems.com
}

\begin{abstract}
Realistic simulation environments play a vital role in the effective training of traffic controllers to respond to large-scale events such as natural disasters or terrorist threats. BAE SYSTEMS has developed a training environment that comprises of: a physical traffic control centre environment, a 3D visualisation and a traffic behaviour model. In this paper, we describe how an agent-based approach has been essential in the development of the traffic operator training environment, especially for constructing the required behavioural models. The simulator has been applied to an evacuation scenario, for which an agent-based model has been developed which models a variety of relevant driver evacuation behaviours. These unusual behaviours have been observed occurring in real-life evacuations but to date have not been incorporated in traffic simulators. In addition, our agent-based approach includes flexibility within the simulator to respond to the variety of decisions traffic controllers can make, as well as achieving a strong degree of control for the scenario manager.
\end{abstract}

Keywords-traffic simulation; traffic operators; agent-based simulation; evacuation simulation;

\section{INTRODUCTION}

Preparing traffic controllers to respond to large-scale events, such as evacuations, requires the development of realistic training environments. These environments need to be easily configurable and controllable by the scenario manager both prior to training and in real-time during training. Within the environment, the traffic controllers should be free to respond to the scenario as they see fit, and thus it is essential that the simulator is able to model the response of traffic to novel decisions made by the traffic controllers. The simulation must then respond realistically to the interventions of the traffic controller, and must realistically model the driver's behaviours which, in an evacuation scenario, including the unusual and exaggerated behaviours that drivers can exhibit due to increase levels of danger and panic. Similarly, it is important that the traffic controller environment and the visual representations of the behaviours of the vehicles be as realistic as possible. For this purpose, the traffic control centre training environment in development at BAE SYSTEMS includes a visualisation studio which provides an immersive physical environment and back-end which produces a realistic simulation of traffic behaviours and responds to the decisions of the traffic controllers. The simulation is supported by a multi-core and multi-processor server which is responsible for creating realistic behaviours in the vehicles.

In more detail, within an evacuation scenario the operators are able to make a number of decisions that affect the behaviours of the traffic including informing the drivers through the use of variable message systems, variable speed limits and radio announcements. The controllers may also exert control over the flow of traffic using road blocks, traffic police and control over the traffic lights through urban traffic control. The simulation framework is thus required to have the flexibility to include all of these types of decisions and crucially, the simulated traffic must respond to them in realistic ways in real-time. The environment must also include corresponding behaviours and driver responses to car accidents and cars breaking down, which have occurred either due to behaviours within the simulation or have been selected to occur by scenario managers. In addition, the simulation must be able to reproduce the unusual driver behaviours observed in real-life evacuations such as herdingtype behaviours. Studies into real-life evacuations have revealed two patterns of behaviour which can be said to be of a herding-type: when drivers perceive some degree of physical danger, they have been observed to choose routes similar to those of others, so as to avoid being isolated [1], [2], and in the high risk and highly uncertain circumstances of an evacuation an individual places enormous trust in the collective knowledge of others, leading to a tendency to almost blindly follow others [3]. Thus, these two unusual behaviours can be represent as two desires of a driver: the desire to be with others and the desire to follow others.

To date, evacuation modelling has been carried out using existing traffic simulators such as MATSIM [4] and PARAMICS [5]. Agent-based modelling techniques have been applied to, or are used in, many of these simulators, which has enabled the observation of the collective behaviour resulting from actions and interactions of individual vehicles during an evacuation. However, the behavioural models offered by traffic simulators are often limited and do not incorporated the unusual behaviours observed within real evacuations, to the extent that their use for disaster management simulation is significantly impaired [6], [7]. 
An additional limitation of existing simulators is that there are no examples of these traffic simulators being used to create a real-time evacuation simulation, in which the traffic controllers can dynamically make decisions, as they would in a real-life evacuation. Due to the nature of traffic simulators, it has been stated that current simulators are inadequate to simulate real-time environments [8]. Traffic simulators often rely on high-level generalised models of traffic behaviour which obeys behaviours believed to be overall correct but are unable to adequately model responses to real-time changes in the environment. This is in contrast to the traffic behaviours used within driving simulators, such as ARCHSIM, in which agent-based techniques can provide simulated vehicles with real-time behavioural responses to the actions and inputs of human beings and thus can provide realistic driver behaviours for potentially any situation [9]. These simulators, however, are not designed for the largescale scenarios required for evacuation simulation.

To address these shortcomings, in this paper we describe our work applying an agent-based approach for modelling the behaviours of the simulated vehicles within a simulated traffic control centre environment. Using our route choice forces model we can simulate the unusual evacuation behaviours as well as plausible driver responses to decisions made by the traffic controllers [10]. Each vehicle is represented by an agent, with particular goals which they are attempting to achieve, such as following an evacuation route to a safe location. The agents are situated within a simulated evacuation environment which includes the road network and the multitude of actors within the road network, such as variable message systems. Our model is based on the social forces model from crowd modelling in which each of the goals or desires is represented by a force acting upon the agent [11]. For the evacuation scenario, two forces are defined which represent the two unusual desires characteristic of evacuation behaviour: the desire to be with others and the desire to follow others. These two forces are in addition to a force representing the desire to travel the quickest route to their destination. The forces dynamically change depending on the actions of the traffic controllers and therefore provide dynamic behaviours for the agents. In addition to our behavioural model and in order to provide the level of realism required for training the simulator framework includes a physical representation of the traffic control centre and a 3D visualiser is used to reproduce the CCTV feeds traffic controllers will use to find out the state of the road network. Thus, in this paper we identify how the use of agents addresses the issues particular to training traffic operators to respond to large-scale events.

\section{SimUlation FrameWORK}

The simulator framework is made up of three components: the traffic control centre environment, the $3 \mathrm{D}$ visualiser

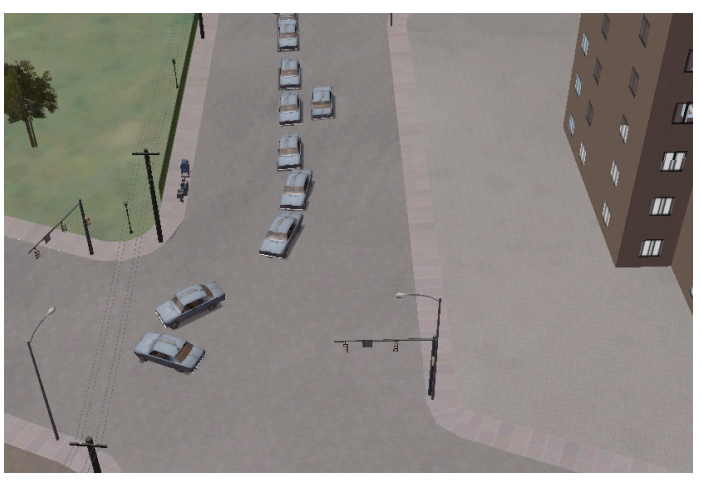

Figure 1: 3D visualiser demonstrating the simulated feed from a CCTV camera

engine and the underlying agent-based traffic behaviour model.

\section{A. Traffic control centre environment}

In order to provide the traffic controllers with an environment comparable to that of a real-life traffic control centre, a physical replication of the traffic control centre is constructed. The environment provides the traffic controllers with the same control over the road networks as in real-life, using similar software and decision support systems. The software provides the controllers with information about the current state of the road network through simulated video feeds from CCTV cameras and vehicle counts on roads. Within our virtual environment, the current state of the traffic is determined through the traffic behaviour model and the $3 \mathrm{D}$ visualiser.

\section{B. $3 D$ visualiser engine}

The visualisation framework is handled by MÄK Technologies' VR-Forces which produces a 3D visualisation of a selected area, in addition to providing a basic physics engine to add to the realism. This $3 \mathrm{D}$ visualisation is used to produce CCTV footage for the traffic controllers, as shown in Figure 1. This provides the primary output from the simulation to the traffic controllers so that they can observe how their actions are affecting traffic. This software also provides the parts of the simulation framework which update the agents, keep track of the time and splits the simulation across multiple processors.

\section{Agent-based traffic behaviour model}

Within the simulator, each vehicle and its respective driver are represented by an agent that provides an individual behavioural model. The agents are proactive in achieving their specific goals via the creation of plans, and reactive to changes in the environment. Their exact behaviours are dependent on their type and "personality", which determines such traits such as whether they are a cautious or an aggressive driver, by the use of parameters such as their maximum 


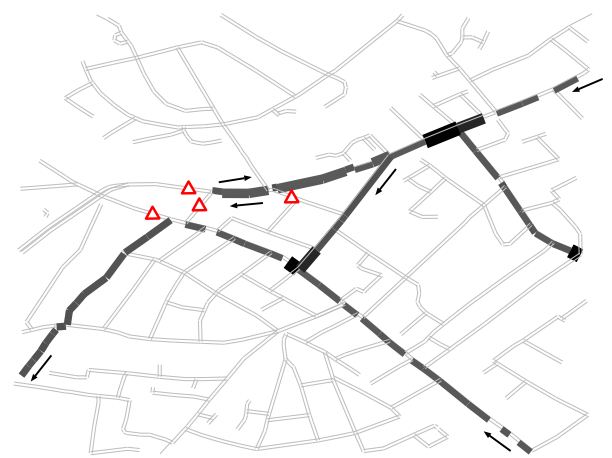

(a) Without modelling unusual behaviours

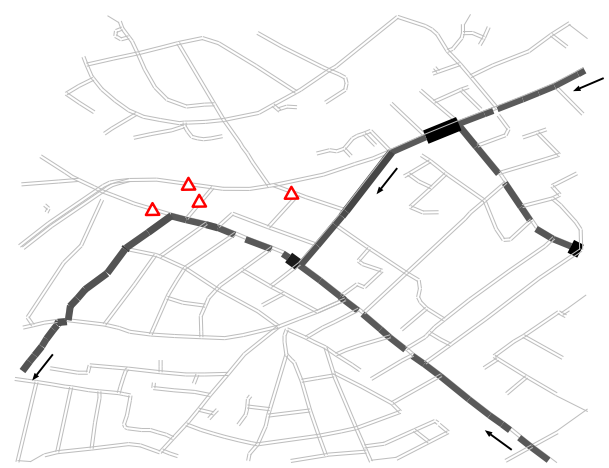

(b) With modelling unusual behaviours

Figure 2: Traffic route choice example

speed. Within the evacuation scenario, their primary goal is to leave the area. The individual nature of the agents means that scenario managers can alter the behaviour of specific drivers in the simulator, for instance by changing their personality type or choosing for their car to breakdown.

The environment is defined as part of the evacuation scenario, which includes the road network to be used, as well as the types of agent to be simulated and the defined events which are to occur at certain points in time, such as accidents. The road network is defined as a set of roads connected by a set of junctions which have a spatial location and the roads have a length, which they agent gains knowledge of when they observe their environment. Additionally, a variety of other environmental actors can be modelled by agents, including the VMS, variable speed limits, traffic lights and radio announcements. This provides a common interface for them, so that they can be represented using the $3 \mathrm{D}$ visualiser and also have their behaviours assigned and configured individually. Alterations to the environment can occur either by the traffic controllers, when they decide to set up a road block, or by the scenario manager, who, for instance, can alter the road network to represent a block road due to an accident.

Within the traffic control centre environment the decisions of the traffic operators exert most control over the strategic behaviour of the drivers such as their choice of route. The tactical behaviours of the driver, such as their exact position on the road, are harder for the traffic operators to influence and require more processing power per agent to simulate. Thus only areas which require a fine level of detail, such as those to be observed by the controllers via CCTV camera or areas in which an accident is to be simulated, are the drivers modelled at this level of accuracy. In other areas the roads are modelled as FIFO buffers with certain restrictions on the entry and exit rates and therefore only which road an agent is on modelled and not the position of the agent on the road, a model based on the agent-based MATSIM traffic simulator [12]. Where the tactical behaviours are being modelled, the agents are updated at regular intervals by the simulation framework, whereas at the strategic level an event-driven approach is used with the agent's behaviour being updated once they reach a junction. At the tactical level the agent has multiple goals such as travelling to the next junction in their route, avoiding over cars including cars involved in accidents, and overtaking in order to advance towards at their desired speed. Once the agent has decided which direction and speed they wish to travel at, this information is then sent to the physics engine which in turn sends it to the 3D visualiser. This adds the required level of realism to create a plausible CCTV camera feed.

At the strategic behaviour level the agents must make a choice about their route to travel, once they reach a junction. Within this simulation the route choice behaviours are provided by our route choice forces model which is based on techniques used in crowd modelling [10]. The use of our agent-based model allows for the explicit inclusion of the more unusual evacuation behaviours in addition to providing the agents with a dynamic way to respond to changes in their environment. Similar to the social forces model, within our model each different desire a driver has is modelled as a force which acts upon the decisionmaking of the agent [11]. Three forces are defined as part of a drivers evacuation behaviour: the desire to evacuate via the quickest route, the desire to be with others, and the desire to follow others. The forces change dynamically as the environment changes depending on the actions of the traffic controllers. These forces depend on the agent's observations of the current environment either myopically or through information gathered from other sources, such as radio announcements. These forces also come from an agent's historic knowledge of an area, which for instances, allows them to know where others are likely to be and therefore attempt to plan routes where they are not isolated from others and fulfil their desire to be with others.

Figure 2 and Figure 3 demonstrate our agent-based behaviour model in use. Figure 2 compares our model to another simpler behaviour model. In this example the scenario managers have decided to block four roads. Figure 2(a) models driver behaviour using only a shortest route algorithm, in which once the agents discovering a blockage 


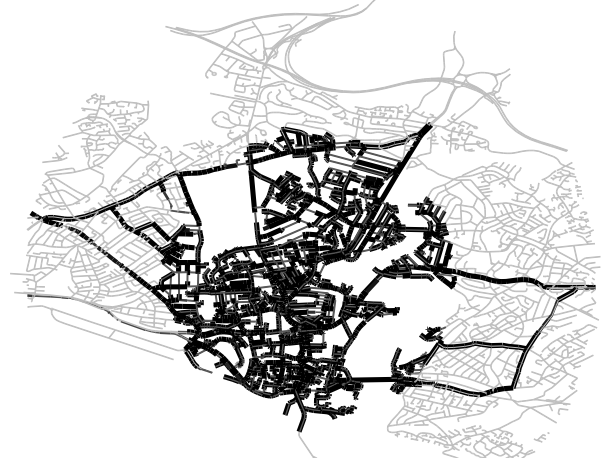

Figure 3: Demonstration of herding behaviour in Southampton, UK

they simply select the next shortest route to their evacuation destination. In Figure 2(b) behaviours are modelling using our route choice forces model. Here the more complex behaviours allowed by our agent-based behavioural model can be observed. The desire to follow others means they select a route in which they dynamically observe the behaviours of others in order to plan their route. Thus, the agents no longer drive up to the blockage and instead follow each other to a different exit. Figure 3 shows large-scale herding behaviour occurring in during the evacuation of Southampton, UK, as simulated by our route choice forces model. The diagram identifies the routes being used by evacuees and it can be seen that driver behaviour has led to only a few evacuation routes being used, with drivers choosing to join others in their route out of town, in order to avoid being isolated.

\section{CONCLUSION}

The benefits of applying an agent-based approach to creating a traffic controller training environment have been identified as flexibility in the model to respond to the decisions of the traffic controllers and scenario managers and more advance behavioural models which represent the more unusual behaviours commonly observed in evacuations. The agents are not controlled by a set of formulas defined at a macroscopic level and instead observe the environment and respond appropriately to fulfil their goals, this way they have the flexibility to respond to any changes and decisions the traffic controllers might make. Additionally, the use of agents has allowed the unusual driver behaviours that occur in reallife evacuations to be adequately simulated, so that traffic controllers can learn to respond to them.

Using an agent-based approach, behavioural models can be more rapidly developed and configured more intuitively as they are modular and based from the driver's point-ofview. Thus the approach provides a convenient way to add novel driver behaviours in order to effectively train traffic operators to the variety of behaviours observed in different scenarios. The approach also provides a simple interface to the visualiser and physics engine. Thus multiple levels of fidelity can be used in the simulator whilst still retaining individual modelling of vehicles.

Further development on the simulator involves the further development of the agent behavioural models. The agent based approached allows for variable driver knowledge of the environment which can be used model a driver's unfamiliarity with an area, which can be occur during an evacuation if a driver follows a prescribed exit route. Individual driver compliance to the suggest evacuation routes and diversions can also be modelled using an agent based approach.

\section{REFERENCES}

[1] K. Dow and S. Cutter, "Emerging hurricane evacuation issues: Hurricane Floyd and South Carolina," Natural Hazards Review, vol. 3, no. 1, pp. 12-18, 2002.

[2] B. Wolshon, "Empirical characterization of mass evacuation traffic flow," Transportation Research Record: Journal of the Transportation Research Board, vol. 2041, pp. 38-48, 2008.

[3] X. Pan, C. Han, K. Dauber, and K. Law, "A multi-agent based framework for the simulation of human and social behaviors during emergency evacuations," AI \& Society, vol. 22, no. 2, pp. 113-132, 2007.

[4] G. Lämmel, M. Rieser, and K. Nagel, "Large scale microscopic evacuation simulation," in Pedestrian and Evacuation Dynamics, Proceedings of the 4th International Conference. Wuppertal, Berlin: Springer, 2008, pp. 547-553.

[5] X. Chen and F. Zhan, "Agent-based modelling and simulation of urban evacuation: Relative effectiveness of simultaneous and staged evacuation strategies," Journal of the Operational Research Society, vol. 59, no. 1, pp. 25-33, 2006.

[6] R. Church and R. Sexton, "Modeling small area evacuation: Can existing transportation infrastructure impede public safety?" California Dept. of Transportation, Tech. Rep., 2002.

[7] R. Alsnih and P. Stopher, "Review of procedures associated with devising emergency evacuation plans," Transportation Research Record: Journal of the Transportation Research Board, vol. 1865, pp. 89-97, 2004.

[8] J. Olstam, J. Lundgren, M. Adlers, and P. Matstoms, “A framework for simulation of surrounding vehicles in driving simulators," ACM Transactions on Modeling and Computer Simulation, vol. 18, no. 3, pp. 1-24, 2008.

[9] A. Champion, S. Éspié, and J. Auberlet, "Behavioral road traffic simulation with ARCHISIM," in Summer Computer Simulation Conference. Society for Computer Simulation International; 1998, 2001, pp. 359-364.

[10] D. Handford and A. Rogers, "Modelling driver interdependent behaviour in agent-based traffic simulations for disaster management," in The Ninth Int. Conference on Practical Applications of Agents and Multi-Agent Systems (to appear), April 2011. [Online]. Available: http://eprints.ecs.soton.ac.uk/21910/

[11] D. Helbing, I. Farkas, and T. Vicsek, "Simulating dynamical features of escape panic," Nature, vol. 407, no. 6803, pp. 487-490, 2000.

[12] N. Cetin and K. Nagel, "A large-scale agent-based traffic microsimulation based on queue model," in Proceedings of Swiss Transport Research Conference, 2003, pp. 3-4272. 